\title{
Airway anesthesia with lidocaine for general anesthesia without using neuromuscular blocking agents in a patient with a history of anaphylaxis to rocuronium: a case report
}

\author{
Sung-Mi Ji, Jaegyok Song, Gunhwa Choi \\ Department of Anesthesiology and Pain Medicine, Dankook University College of Medicine, Cheonan, Korea
}

\begin{abstract}
We experienced a case of induction of general anesthesia without using neuromuscular blocking agents (NMBAs) in a 40-year-old woman with a history of anaphylaxis immediately after the administration of anesthetics lidocaine, propofol, and rocuronium to perform endoscopic sinus surgery 2 years before. The skin test showed a positive reaction to rocuronium and cis-atracurium. We induced general anesthesia without using NMBAs after inducing airway anesthesia with lidocaine (transtracheal injection and superior laryngeal nerve block). Deep general anesthesia was maintained with end-tidal $4 \mathrm{vol} \%$ sevoflurane. Hypotension was treated with phenylephrine infusion. The operation condition was excellent, and patient recovered without complications after surgery. Airway anesthesia with local anesthetics may be helpful when we cannot use NMBAs for any reason, including hypersensitivity to NMBA and surgery that needs neuromuscular monitoring.
\end{abstract}

Keywords: Anaphylaxis; Hypersensitivity; Neuromuscular Blocking Agents; Rocuronium.

This is an Open Access article distributed under the terms of the Creative Commons Attribution Non-Commercial License (http://creativecommons.org/licenses/by-nc/4.0/) which permits unrestricted non-commercial use, distribution, and reproduction in any medium, provided the original work is properly cited.

\section{INTRODUCTION}

Perioperative anaphylactic reaction is a rare but life-threatening complication [1-5]. Nearly all kinds of medications and substances used in anesthesia and surgery can cause anaphylaxis, including hypnotic opioids, local anesthetics, colloids, dyes, antibiotics, neuromuscular blocking agents (NMBAs), disinfectants, and latex [4]. NMBAs are a common substance involved in anaphylaxis [5]. Paralysis with NMBA is a major component of general anesthesia that facilitates smooth tracheal intubation; improves the surgical condition, such as muscle relaxation of the surgical field; and protects the patient from injuries caused by unexpected movements during surgery. Patients with a history of anaphylaxis to NMBA can be a challenge to anesthesiologists. The best option is the investigation of safe NMBA through allergic tests [6]. However, if there is no safe, available NMBA, we should consider other options. We experienced a case of induction of general anesthesia with topical and local blocks of the airway without using NMBAs in a 40 -year old woman with a history of anaphylaxis immediately after the administration of anesthetics lidocaine, propofol, and rocuronium. We would like to report this case with a brief review of the literature.

Received: Mar 14, 2020 - Revised: May 15, 2020 - Accepted: May 19, 2020

Corresponding Author: Jaegyok Song, Department of Anesthesiology and Pain Medicine, Dankook University College of Medicine, 119, Dandae-ro, Dongnam-gu,

Cheonan-si, Chungnam 31116, Korea

Tel: +82-41-550-6819 E-mail: drjack@nate.com

Copyright(c) 2020 Journal of Dental Anesthesia and Pain Medicine 
Table 1. Reported nonirritating maximal concentrations for the intraderma test (adapted from [4])

\begin{tabular}{|c|c|c|c|}
\hline \multirow{2}{*}{ Agent } & \multirow{2}{*}{$\begin{array}{l}\text { Undiluted } \\
\text { (mg/mL) }\end{array}$} & \multicolumn{2}{|c|}{ Intradermal Test (mg/mL) } \\
\hline & & Dilution & $\operatorname{Cmax}(\mathrm{mg} / \mathrm{mL})$ \\
\hline Cis-atracurium & 2 & $1 / 100$ & 0.02 \\
\hline Rocuronium & 10 & $1 / 100$ & 0.1 \\
\hline Fentanyl & 0.05 & $1 / 10$ & 0.005 \\
\hline
\end{tabular}

\section{CASE REPORT}

Written consent was obtained from the patient for publication of this report. A 40-year-old woman (American Society of Anesthesiologists class I; weight, $54 \mathrm{~kg}$; height, $161 \mathrm{~cm}$ ) with chronic rhinitis and nasal polyps was scheduled for endoscopic sinus surgery. She had a history of allergic rhinitis, asthma, as well as severe anaphylaxis and cardiac arrest after the induction of general anesthesia with lidocaine, propofol, and rocuronium to perform endoscopic sinus surgery 2 years before. The patient had no related sequelae. Preoperative evaluations, including laboratory tests, electrocardiography, and chest radiography, were unremarkable.

The patient showed no hypersensitivity reactions to propofol or lidocaine in the intradermal skin test before anesthesia. We tested rocuronium and cis-atracurium before the induction of general anesthesia to determine which drug was safe for the patient. She took no antihistamines; however, an intravenous injection of methyl prednisolone $30 \mathrm{mg}$ was administered $3 \mathrm{~h}$ before the test. Patient monitors were instituted, including standard monitors and continuous arterial blood pressure monitoring with radial artery catheter insertion. Subsequently, we performed intradermal skin tests in the ventral area of forearm [4,5]. Fentanyl was diluted to $1 / 10$ (5 $\mu \mathrm{g} / \mathrm{mL}$, Table 1). Both rocuronium and cis-atracurium were diluted to $1 / 100$ (rocuronium $0.1 \mathrm{mg} / \mathrm{mL}$, cis-atracurium $0.02 \mathrm{mg} / \mathrm{mL}$ ) [5]. Each test drug and normal saline (negative control) $0.05 \mathrm{~mL}$ were intradermally injected, and a 2-mm-sized bleb was made. Distance between those skin test sites were more than
Table 2. Results of the skin test to fentanyl, saline, rocuronium, and cis-atracurium

\begin{tabular}{cccc}
\hline Agent & Bleb $(\mathrm{mm})$ & Wheal $(\mathrm{mm})$ & Flare $(\mathrm{mm})$ \\
\hline Normal Saline & & $0 \times 0$ & $0 \times 0$ \\
Cis-atracurium & $2 \times 2$ & $3 \times 4$ & $11 \times 15$ \\
Rocuronium & $2 \times 3$ & $9 \times 8$ & $24 \times 15$ \\
Fentanyl & & $1 \times 1$ & $2 \times 3$ \\
\hline
\end{tabular}

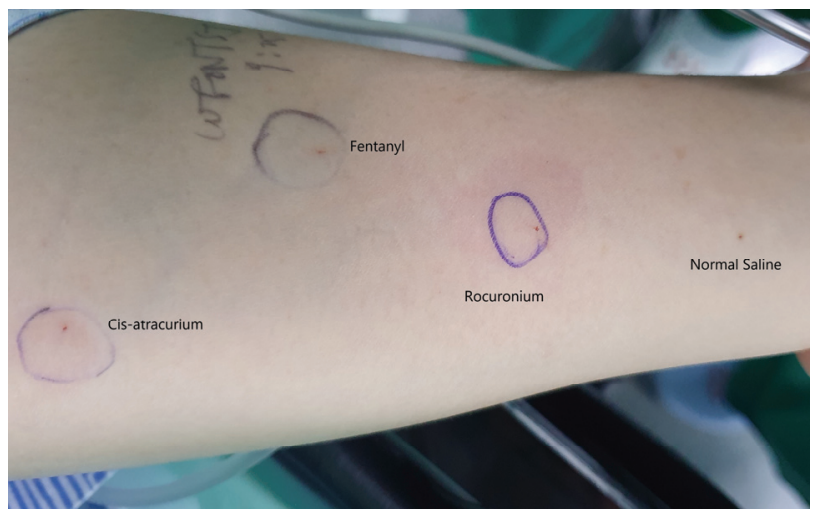

Fig. 1. Result of the intradermal skin test. The intradermal skin test shows a positive response to rocuronium.

$5 \mathrm{~cm}$. Skin reactions were checked $20 \mathrm{~min}$ after the injection. Fentanyl and cis-atracurium showed a negative reaction, but rocuronium showed a positive reaction (Fig. 1, Table 2). However, we did not have histamine or codeine for a positive control at the time and could not confirm the safety of cis-atracurium. Vecuronium was also temporarily unavailable at that time, so we decided to perform deep general anesthesia without NMBAs.

We discussed the patient's situation with her and obtained written consent for the procedure. We induced topical and local anesthesia to the airway with lidocaine to decrease the airway reflex to endotracheal intubation and airway irritation during surgery (Fig. 2, 3). While performing preoxygenation, $50 \mu \mathrm{g}$ fentanyl was intravenously injected, and $5 \mathrm{~min}$ later, skin on the cricothyroid membrane was sterilized with an alcohol sponge. Further, the skin was anesthetized with $2 \%$ lidocaine infiltration. Subsequently, the cricothyroid membrane was punctured with a 22-G intravenous needle, and Teflon catheter was gently inserted into the trachea after confirming air aspiration. After confirming the 


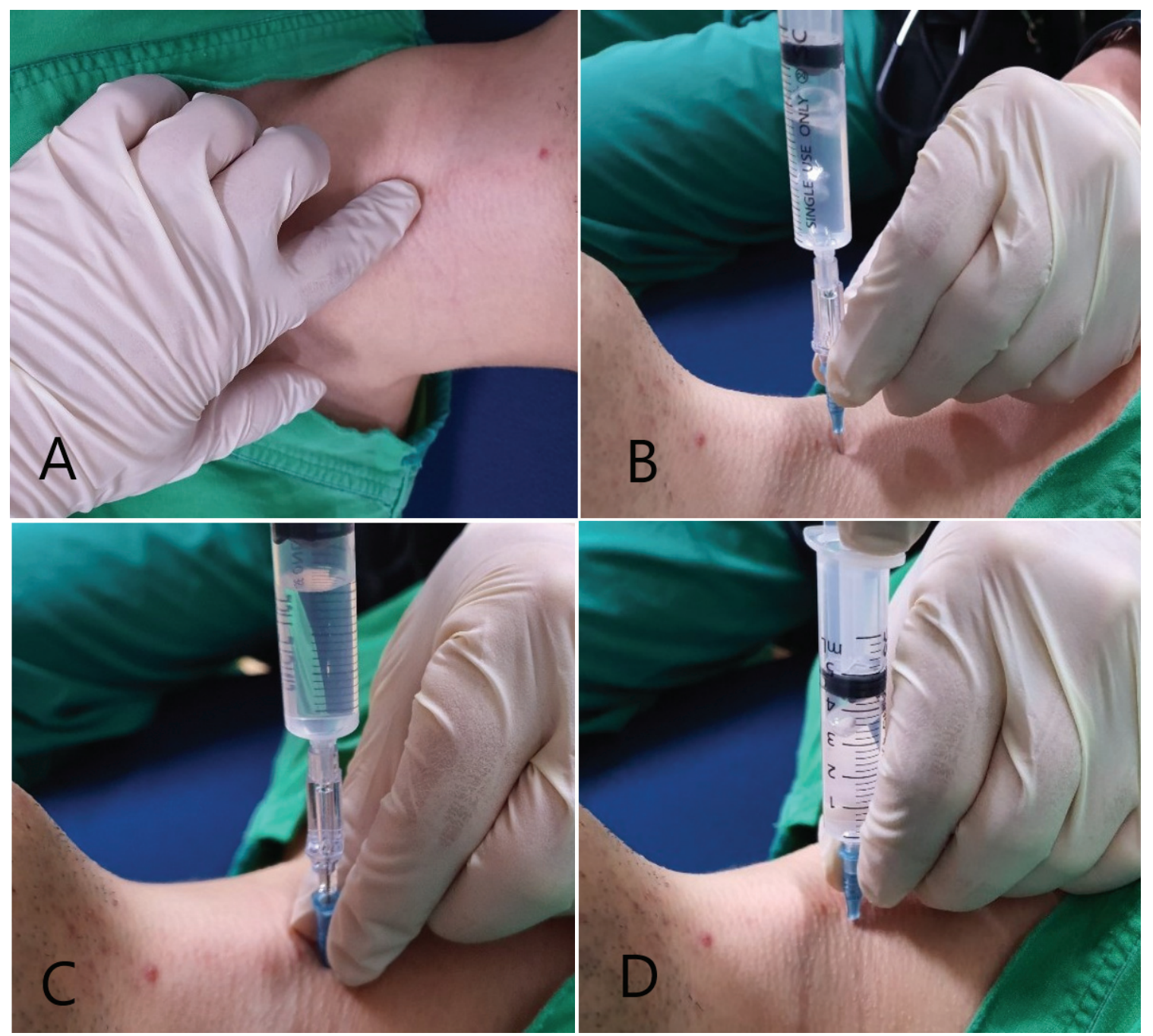

Fig. 2. Transtracheal injection procedure. A: Find the cricothyroid membrane between the thyroid cartilage and the cricoid cartilage. B: Puncture the cricothyroid membrane with a $22-\mathrm{G}$ needle. Note air aspiration. C: Advance the Teflon catheter into the trachea. D: Connect $4 \mathrm{~mL}$ of $2 \%$ lidocaine, and inject it after confirming air aspiration.

catheter tip site through air aspiration, $4 \mathrm{~mL}$ of $4 \%$ lidocaine was injected into the trachea. Subsequently, the catheter was removed. After three rounds of vigorous coughing, the patient relaxed. Topical anesthesia in the oropharyngeal area was performed with a lidocaine spray. General anesthesia was induced with an intravenous injection of $60 \mathrm{mg}$ lidocaine and $100 \mathrm{mg}$ propofol. Mask ventilation was performed for $5 \mathrm{~min}$ with $100 \%$ oxygen and 5 vol\% sevoflurane. During mask ventilation, bilateral superior laryngeal nerve blocks were performed using $2 \mathrm{~mL}$ of $2 \%$ lidocaine injected on each side of the superior cornu of thyroid cartilage. After confirming no more self-respiration, the patient was intubated with conventional direct laryngoscopy. The intubation condition was good, and there was no coughing or other movements. After the induction of anesthesia, general anesthesia was maintained with sevoflurane (End-tidal concentration was continued with 4 vol\%.) to induce deep anesthesia without using NMBAs, and phenylephrine was continuously infused $(0.5 \sim 1 \mu \mathrm{g} / \mathrm{kg} / \mathrm{min})$ to treat mild hypotension. The surgery was performed successfully, and operation time was $1 \mathrm{~h}$ and $42 \mathrm{~min}$. There were no episodes of hemodynamic instability during the surgery. The operation field condition was very good, and there were no movements during the surgery. Her bispectral index score was between 19 and 32 during the surgery. 


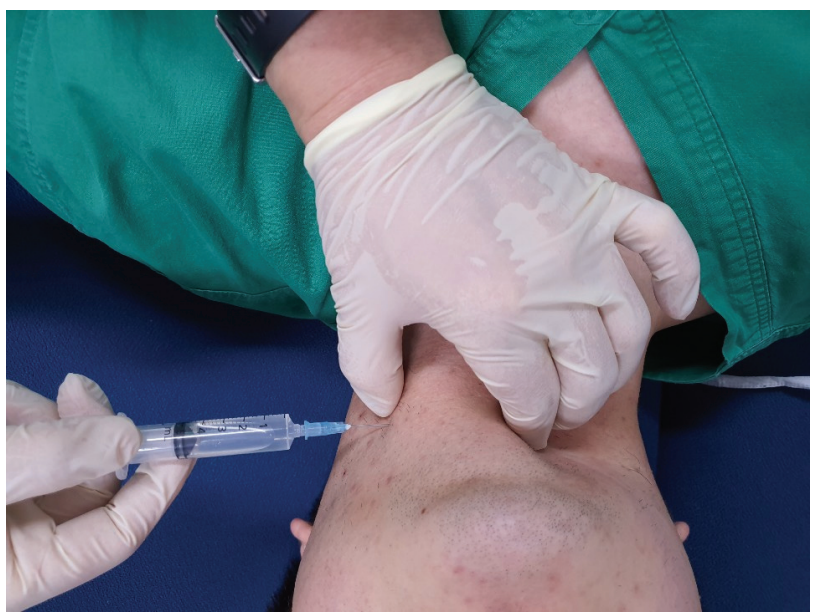

Fig. 3. Superior laryngeal nerve block on the left side. Grab the superior cornu of the thyroid cartilage with the index finger and thumb and push the thyroid cartilage to the lateral side. With a 23-G needle, the operator walks off the superior cornu of the thyroid cartilage until the thyrohyoid membrane is pierced and injects $2 \sim 3 \mathrm{~mL}$ of $2 \%$ lidocaine.

The surgeon felt no difference from patients who are under relaxation with NMBAs. The present patient successfully recovered $20 \mathrm{~min}$ after the cessation of sevoflurane without complications and was extubated. She was then transferred to the post-anesthesia care unit. Finally, she was discharged the next day after surgery without complications.

\section{DISCUSSION}

What are the options for anesthetic induction in patients with drug allergies? The optimal strategy is to determine the causative drug and avoid administering it. First, we should review the patient's past history, which may reveal or narrow the range of drugs. Although several issues remain unestablished, the diagnostic method of choice is the skin test [5]. In this case, rocuronium showed a definite positive sign, and cis-atracurium was negative. The safety of cis-atracurium could not be confirmed with this result. Cross-sensitivity is estimated to be $60 \% \sim 70 \%$ for NMBAs [2]. Thacker et al. [7] strongly recommended performing intradermal tests for patients with a history of hypersensitivity to NMBAs. The pattern of crosssensitivity may vary across NMBAs. Those with similar structures show greater cross-sensitivity. For example, pancuronium and vecuronium, succinylcholine and gallamine, and atracurium and cis-atracurium often showed cross-sensitivity, and $7 \%$ of the drugs show cross-sensitivity to all NMBAs [2]. Fisher et al. [8] reported one patient with a history of hypersensitivity and attributed it to alcuronium. The patient had a second reaction to pancuronium, which had shown a positive skin test. They suggested using no NMBAs in order to avoid risks of cross-sensitivity. The patient was treated with methylprednisolone $3 \mathrm{~h}$ before surgery, but systemic corticosteroids used for a short period and in low to moderate doses do not decrease skin test reactivity [9]. We decided to perform the anesthesia without using NMBAs.

Variable methods and drugs have been tried when performing induction and management of general anesthesia without NMBAs by many anesthesiologists [10-13]. The most common concepts of those methods were using deep anesthesia with volatile anesthetics, propofol, opioids, and dexmedetomidine. However, deep anesthesia enough to blunt airway reflexes may cause hemodynamic instability during the surgery. Rajan et al. [10] reported six cases of sevoflurane-induced deep general anesthesia without using NMBAs or intravenous general anesthetics, and two patients with a history of hypertension showed persistent hypotension and required epinephrine infusion. In our case, the surgery was performed in the facial area and did not require abdominal muscle relaxation. However, frequent irritation to the airway was expected. We thought that airway anesthesia with local anesthetics will decrease the airway reflex that may facilitate tracheal intubation and decrease the need for excessive deep anesthesia during the surgery. We administered a transtracheal injection of lidocaine and the superior laryngeal nerve block. There was no movement or airway reflex during the surgery. After the end of surgery (approximately $2 \mathrm{~h}$ after the local block of the airway), the airway protective reflex was intact when recovering from anesthesia. Besides anaphylaxis to NMBAs, there are surgeries that need neuromuscular 
monitoring during the procedures, including parotidectomy, ear surgery, and various neurosurgeries. Airway blocks with local anesthetics may be a good option in these situations. Rajan et al. [14] reported that a transtracheal lidocaine injection showed a similar effect as propofol infusion and recommended transtracheal lidocaine injection as a successful alternative to propofol infusion because it is cheaper and safe.

Airway anesthesia with local anesthetics has some benefits over deep general anesthesia with a high dose of general anesthetics or multiple drug combinations. First, direct blunting of airway reflexes with local anesthetics that follow hemodynamic stability and decrease the requirement of general anesthetics. Second, it decreases the need for excessive deep anesthesia that can induce delay in recovery from general anesthesia. Third, it may decrease medical costs related to drugs and the equipment for continuous infusion.

However, there are some drawbacks to this method. If the surgery ends too early, remaining airway anesthesia may increase the risk of aspiration pneumonia. If the patient is not cooperative, it is difficult to perform this procedure while the patient is awake. If the surgery is longer than $2 \mathrm{~h}$, the effect may not be effective enough. This method may not be helpful if the surgery is laparotomy, which needs abdominal muscle relaxation.

In conclusion, we experienced a case of a patient who had hypersensitivity to NMBAs, and successful anesthetic management without NMBAs was performed with airway anesthesia with lidocaine. This method may be helpful when we cannot use NMBAs for any reason, including hypersensitivity to NMBA and surgery that needs neuromuscular monitoring.

\section{AUHOR ORCIIS}

Sung-Mi Ji: https://orcid.org/0000-0002-9633-8086

Jaegyok Song: https://orcid.org/0000-0002-4727-6296

Gunhwa Choi: https://orcid.org/0000-0003-1554-1355
AUHOR BONIRIBUIOHS

Sung-Mi Ji: Writing - review \& editing

Jaegyok Song: Conceptualization, Data curation, Project

administration, Supervision, Writing - review \& editing

Gunhwa Choi: Writing - original draft

CONFLICT OF INTEREST: There are no financial or other issues that might lead to conflict of interest.

\section{REFERENCES}

1. Brereton A, Russell WJ. Anaphylaxis to muscle relaxants: an audit of ten years of allergy testing at the Royal Adelaide Hospital. Anaesth Intensive Care 2012; 40: 861-6.

2. Volcheck GW, Hepner DL. Identification and management of perioperative anaphylaxis. J Allergy Clin Immunol Pract 2019; 7: 2134-42.

3. Jang YH, Kim SG, Son YH, Park JM. Rocuronium bromide induced anaphylaxis in a child -a case report-. Korean J Anesthesiol 2010; 59: 411.

4. Mertes PM, Lambert M, Gueant-Rodriguez RM, AimoneGastin I, Mouton-Faivre C, Moneret-Vautrin DA, et al. Perioperative anaphylaxis. Immunol Allergy Clin North Am 2009; 29: 429-51.

5. Ebo DG, Fisher MM, Hagendorens MM, Bridts $\mathrm{CH}$, Stevens WJ. Anaphylaxis during anaesthesia: diagnostic approach. Allergy 2007; 62: 471-87.

6. Pedersen AF, Green S, Rose MA. Failure to investigate anaesthetic anaphylaxis resulting in a preventable second anaphylactic reaction. Anaesth Intensive Care 2012; 40: 1053-5.

7. Thacker MA, Davis FM. Subsequent general anaesthesia in patients with a history of previous anaphylactoid/ anaphylactic reaction to muscle relaxant. Anaesth Intensive Care 1999; 27: 190-3.

8. Fisher MM, Bowey CJ. Intradermal compared with prick testing in the diagnosis of anaesthetic allergy. Br J Anaesth 1997; 79: 59-63.

9. Des Roches A, Paradis L, Bougeard YH, Godard P, 
Bousquet J, Chanez P. Long-term oral corticosteroid therapy does not alter the results of immediate-type allergy skin prick tests. J Allergy Clin Immunol 1996; 98: 522-7.

10. Rajan S, Mathew J, Tosh P, Sudevan M. Safety of inhalational anesthesia in patients with multiple drug allergies presenting for major surgeries under general anesthesia. Anesth Essays Res 2019; 13: 259-63.

11. Ono AH, Moura TR, Goveia CS, Guimaraes GMN, de Araujo Ladeira LC, da Silva HBG. ED50 of remifentanil for providing excellent intubating conditions when co-administered with a single standard dose of propofol without the use of muscle relaxants in children: dosefinding clinical trial. J Anesth 2018; 32: 493-8.
12. Abdelhalim AA, Maghraby HH, ElZoughari IA, AlZahrani TA, Moustafa MS, Alfassih KM, et al. Using fentanyl and propofol for tracheal intubation during sevoflurane induction without muscle relaxants in children: a randomized prospective study. Saudi J Anaesth 2017; 11: 312-8.

13. Wei L, Deng X, Sui J, Wang L, Liu J. Dexmedetomidine improves intubating conditions without muscle relaxants in children after induction with propofol and remifentanil. Anesth Analg 2015; 121: 785-90.

14. Rajan S, Puthenveettil N, Paul J. Transtracheal lidocaine: an alternative to intraoperative propofol infusion when muscle relaxants are not used. J Anaesthesiol Clin Pharmacol 2014; 30: 199-202. 